\title{
MODELING AND SENSITIVITY ANALYSIS OF RESERVATION BASED USAP HARD SCHEDULING UNICAST TRAFFIC IN MANETS
}

\author{
Senni Perumal*, Vahid Tabatabaee ${ }^{\dagger}$, John S. Baras*, Charles J. Graff ${ }^{\ddagger}$ and David G. Yee ${ }^{\ddagger}$ \\ *AIMS Inc., 6213 Executive Blvd., Rockville, Maryland 20852 \\ Email: senni.perumal@aims-sys.com,john.baras@aims-sys.com \\ $\dagger$ Institute for Systems Research, University of Maryland, College Park, Maryland 20742 \\ Email: vahidt@umd.edu \\ ‡US Army CERDEC S\&TCD, AMSRD-CER-ST-WL-NS, Fort Monmouth, NJ 07703 \\ Email: Charles.Graff@us.army.mil, David.Yee2@us.army.mil
}

\begin{abstract}
In this paper, we develop and evaluate models to estimate and optimize various performance metrics for unicast traffic using the hard scheduling (virtual circuit connectionoriented) mode of the reservation based protocol USAP in mobile ad-hoc wireless networks (MANETS). USAP (Unifying Slot Assignment Protocol) is a dynamic distributed resource allocation protocol for MANETs. We model USAP hard scheduling mode using loss network models along with reduced link capacity that couple physical, MAC and routing layers effects. The key effect of multiuser interference on a link is modeled via reduced link capacity where the effects of transmissions and receptions in the link neighborhood are modeled using USAP reservation rules. We compare our results with simulation and obtain good results using our loss network models but with reduced link capacity distribution obtained by simulation. We also compute the throughput sensitivities with respect to network parameters and use these sensitivities to improve network performance.
\end{abstract}

\section{INTRODUCTION}

Unifying Slot Assignment Protocol [1] is a particular reservation based Medium Access Control (MAC) protocol that divides the communication channel into orthogonal cells with a portion for management traffic and the rest for user traffic. The USAP protocol operates in two modes: hard scheduling mode (virtual circuit connection-oriented mode) where nodes reserve a session's link capacity end-to-end over the entire path; and soft scheduling mode (datagram scheduling) where nodes perform per-hop scheduling of links for single packets after the packet's arrival at the node.

In this paper, we develop models for USAP hard scheduling mode and use them to both approximate the performance of a MANET and to optimize network performance. Our approach to performance evaluation and optimization is based on fixed point methods and reduced load approximations for loss network models. Loss network models [2] were originally used to compute blocking probabilities in circuit switched networks [3] and later were extended to model and design ATM networks [4]-[7]. The main challenge in developing loss network models for wireless networks is coupling between wireless links. This coupling is due to sharing of wireless medium between a node and its neighbors, resulting in a node's average link capacity to be dependent on its neighborhood traffic. We model this effect via reduced average link capacity calculated using USAP reservation rules and traffic among neighboring nodes.

We assume we know the exogenous traffic rate for each source-destination pair and use multiple paths with a set of routing probabilities to forward traffic between a source and destination. The reduced load loss network model coupled with the reduced wireless link capacity estimation model and multiple path routing give us a set of a non-linear equations that are run iteratively to obtain a fixed point estimate of performance metrics like blocking probability and throughput. We then use the reduced load loss network model to calculate throughput sensitivities which are used to compute the optimal load distribution among multiple paths to maximize network throughput.

The paper is organized as follows. Section II introduces the USAP protocol. Section III describes our fixed point models for USAP Hard Scheduling. Section IV discusses how we calculate throughput sensitivities and use them to maximize network throughput by computing optimal load distribution among multiple paths of a source-destination connection. Finally, in section $\mathrm{V}$ we present results of our USAP Hard Scheduling model and compare them against simulation. Individual connection throughput shows good match with simulation when we use the reduced load loss network model but with reduced link capacity distribution obtained from simulation. Hence we can optimize network throughput using throughput sensitivities obtained from reduced load loss network models along with reduced link capacity distribution obtained from simulation. 


\section{USAP MAC PROTOCOL}

USAP is the distributed resource allocation protocol used in Wireless-wideband Networking Engine (WNE: Rockwell Collins' tactical battlefield wireless ad-hoc protocol suite). WNE channel access is also adapted to the Mobile Data Link (MDL) layer of the Joint Tactical Radio System (JTRS) Wideband Networking Waveform (WNW) [8].

USAP protocol allows a transmitter to choose slots from the pool of unassigned slots in its neighborhood, coordinate the announcement and confirmation of this assignment with neighboring nodes upto two hops away, and detect and resolve conflicting assignments. USAP partitions the channel into time and frequency cells and constructs a periodic communication frame structure (see Fig. 1). Bootstrap minislots are pre-allocated to nodes for exchange of network management information and are used to reserve data channel cells. Broadcast slots support multicast/broadcast data and we do not consider them in our unicast modeling. Reservation slots support unicast data traffic. USAP frame reservation slots consists of $M \times F$ cells, where $M$ is the number of time slots and $\mathrm{F}$ is the number of frequency channels in a frame. Once a cell is assigned to link $(i, j)$, there is no contention as no node transmissions in the twohop neighborhood interfere with link $(i, j)$ transmission. Nodes $i$ and $j$ cannot transmit or receive on any other frequency channel corresponding to that time slot.

In the control channel (bootstrap mini-slots), every node broadcasts the cells that are reserved for transmission and reception by itself and its neighbors. In this way, every node acquires information about the reserved slots in its 2-hop neighborhood. Let $T(l)$ and $R(l)$ be the transmitting and receiving nodes of link $l$ respectively. To avoid collision, cell reservation by node $T(l)$ for transmission to $R(l)$ is based on the following rules: 1) $T(l)$ cannot reserve other cells (with different frequency) on those time slots which already have scheduled incoming or outgoing cell transmissions to and from $T(l)$ and $R(l) ; 2) T(l)$ cannot reserve cells used by incoming call transmissions to the neighbors of $T(l)$; 3) $T(l)$ cannot reserve cells used by outgoing call transmissions from the neighbors of $R(l)$. These rules form the basis for our link capacity approximation described later.

USAP can function under a connection-oriented (hard scheduling) or connection-less (soft scheduling) framework. We model USAP hard scheduling mode where cells are reserved for the entire call duration on all links of the path from the source to destination. The performance metric for the hard scheduling case is the percentage of calls blocked for each connection. A call is blocked if there is not enough available capacity (cells in frame) on all links of the path. There is no significant queuing for the hard scheduling case; hence, delay is not an essential performance metric.

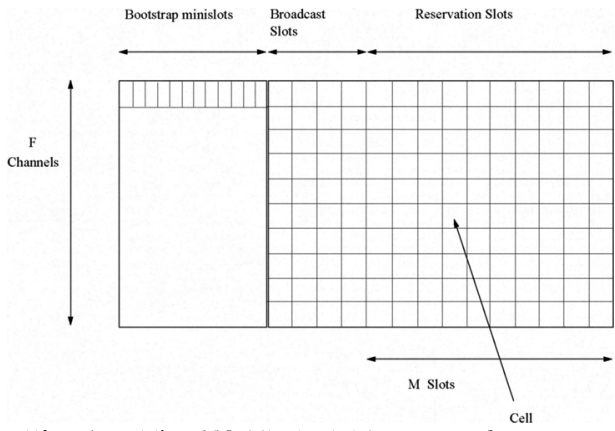

Fig. 1. The USAP TDMA Frame Structure

\section{USAP HARD SCHEDULING MODEL}

We first briefly recollect the key equations in the reduced load loss network approximation for blocking probability computation of an incoming virtual circuit connection in a network. We are given the statistics of all ongoing sourcedestination connections along with the routes assigned to these connections. We assume that calls for route $r$ arrive as a Poisson process with rate $\nu_{r}$ and with mean holding time $1 / \mu_{r}$. The call demand in terms of the number of (reserved) cells per frame is $n_{r}$.

Offered load, $\rho_{l, r}$, of route $r$ calls arriving at link $l$ is reduced due to blocking on other links in the route and is given by

$$
\rho_{l, r}=\frac{\nu_{r}}{\mu_{r}} \prod_{k \in r /\{l\}}\left(1-B_{k, r}\right)
$$

where, $B_{k, r}$ is the probability of blocking a call on link $k$ along route $r$. The blocking probability, $L_{r}$, for a connection traversing route $r$ towards its destination is given by

$$
L_{r}=1-\Pi_{l \in r}\left(1-B_{l, r}\right)
$$

Denote by $Q_{r}\left[C_{l} ; \rho_{r^{\prime}}, r^{\prime} \in R_{l}\right]$ the blocking probability for route $r$ calls on a link $l$ with capacity $C_{l}$ which has a set of routes $R_{l}$ going through it. We have,

$$
Q_{r}\left[C_{l} ; \rho_{r^{\prime}}, r^{\prime} \in R_{l}\right]=1-\sum_{c=0}^{c=C_{l}-n_{r}} q_{C_{l}}(c)
$$

where the $q_{C_{l}}(c)^{\prime} s$, are the probabilities of having $c$ cells occupied on link $l$ with capacity $C_{l}$ (i.e., knapsack occupancy probabilities, chapter 2 of [9]).

In the wireless network case the link capacity is not fixed, and it depends on the number of ongoing connections in the 2-hop neighborhood of the link. For any given value of link capacity $m$, the occupancy probabilities can be computed easily using the standard recursive stochastic knapsack algorithm (chapter 2 of [9]). If we assume that the link $l$ capacity is between $C_{l}^{\min }$ and $C_{l}^{\max }$ with some given probability distribution, we have

$$
B_{l, r}=\sum_{m=C_{l}^{\min }}^{C_{l}^{\max }} \operatorname{Pr}\left[C_{l}=m\right] Q_{r}\left[m ; \rho_{l, r^{\prime}}, r^{\prime} \in R_{l}\right]
$$




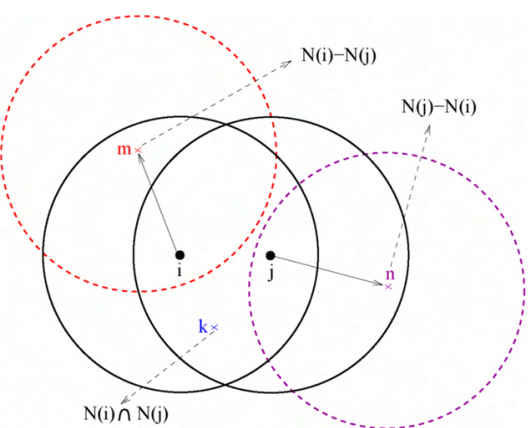

Fig. 2. Neighbors of $i \& j$ : TXs $(i, m) \&(j, n)$ can share same cell

From the occupancy probabilities, we also compute $\eta_{i j}$, the average number of cells reserved by link $l=(i, j)$ :

$$
\eta_{i j}=\sum_{m=C_{l}^{\min }}^{C_{l}^{\max }} \operatorname{Pr}\left[C_{l}=m\right] \sum_{c=0}^{m} c q_{m}(c)
$$

We need to estimate link capacity probabilities $P\left[C_{l}=\right.$ $m$ ] in eqns (4), (5). We describe our methodology for estimating the capacity distribution in the following section.

\section{A. Link Capacity Estimation}

Consider link $l$ with transmitting node $i$ and receiving node $j$. Let $N(i)$ denote the neighbors of node $i$. Neighbors of $i$ and $j$ can be split into the following (see figure 2): neighbors common to $i$ and $j$, i.e., $N(i) \cap N(j)$; neighbors of $i$ hidden from $j$, i.e., $N(i)-N(j)$; and neighbors of $j$ hidden from $i$, i.e., $N(j)-N(i)$. Denote by: $\Gamma_{i}^{T}$ and $\Gamma_{i, X}^{T}$ the average number of reserved slots used by node $i$ in transmitting to all its neighbors and to neighbors in set $X$ respectively; $\Gamma_{i}^{R}$ and $\Gamma_{i, Y}^{R}$ the average number of reserved slots used by $i$ in receiving from all its neighbors and from neighbors in node set $Y$ respectively. Then,

$$
\begin{aligned}
& \Gamma_{i}^{T}=\sum_{k \in N(i)} \eta_{i k}=\Gamma_{i, N(i) \cap N(j)}^{T}+\Gamma_{i, N(i)-N(j)}^{T}+\eta_{i j} \\
& \Gamma_{i}^{R}=\sum_{k \in N(i)} \eta_{k i}=\Gamma_{i, N(i) \cap N(j)}^{R}+\Gamma_{i, N(i)-N(j)}^{R}+\eta_{j i}
\end{aligned}
$$

We calculate $C_{l}^{\min }$ and $C_{l}^{\max }$, a low and high estimate of the number of cells available to link $l$ for sending traffic, by using USAP reservation rules and computing minimum and maximum estimates for the number of slots and cells used by $i, j$, and their neighbors. A slot refers to all the cells (over all frequency channels) at that particular time slot in the frame. Corresponding to the first USAP reservation rule (section II), we denote by $R_{\min }^{1}(i, j)$ and $R_{\max }^{1}(i, j)$ the low and high estimate respectively of the number of time slots used by the nodes $i$ and $j$ to transmit and receive from their neighbors barring the transmission from $i$ to $j$. Corresponding to the second reservation rule, we denote by $R_{\min }^{2}(i, j)$ and $R_{\max }^{2}(i, j)$ the low and high estimate respectively of the number of cells used by the neighbors of $i$ (except $j$ ) to receive traffic from their neighbors except the transmissions from $i$ and $j$ to neighbors of $i$. Similarly corresponding to the third reservation rule, we denote by $R_{\min }^{3}(i, j)$ and $R_{\max }^{3}(i, j)$ the low and high estimate respectively of the number of cells used by the neighbors of $j$ (except $i$ ) to transmit traffic to their neighbors except the transmissions from $j$ 's neighbors to $j$ and $i$. We assume a uniform distribution between $C_{l}^{\min }$ and $C_{l}^{\max }$.

1) Computing $R_{\min }^{1}(i, j)$ and $R_{\max }^{1}(i, j)$ : From the USAP reservation rules and neighbor sets of $i$ and $j$ (figure $2)$, we infer that transmissions between sets $[i \rightarrow N(i)$ $N(j)]$ and $[j \rightarrow N(j)-N(i)]$ can share cells. The same applies to the sets $[N(i)-N(j) \rightarrow i]$ and $[N(j)-N(i) \rightarrow j]$. We also deduce that any transmissions between the sets $[i \rightarrow N(i) \cap N(j)],[N(i) \cap N(j) \rightarrow i],[j \rightarrow N(i) \cap N(j)]$, and $[N(i) \cap N(j) \rightarrow j]$ cannot share cells. Furthermore, when nodes $i$ and $j$ transmit to or receive from a common neighbor $k$ in the set $N(i) \cap N(j)$, then these transmissions or receptions not only cannot share the same cell but also cannot share the same time slot. We consider two cases $F=1$ and $F>1$ when computing $R_{\min }^{1}(i, j)$ and $R_{\max }^{1}(i, j)$.

Case $\mathbf{F}=1$ : Since transmissions $[i \rightarrow N(i)-N(j)]$ and $[j \rightarrow N(j)-N(i)]$ can share cells (with $F=1$, share time slots), the minimum number of slots needed is the maximum of the two. Similar arguments hold for transmissions $[N(i)-N(j) \rightarrow i]$ and $[N(j)-N(i) \rightarrow j]$. Since transmissions between the sets $[i \rightarrow N(i) \cap N(j)],[N(i) \cap$ $N(j) \rightarrow i],[j \rightarrow N(i) \cap N(j)]$, and $[N(i) \cap N(j) \rightarrow j]$ cannot share cells, now with $F=1$, these sets have to be allocated in different time slots. Therefore,

$$
\begin{array}{r}
R_{\min }^{1}(i, j)=\Gamma_{i, N(i) \cap N(j)}^{T}+\Gamma_{i, N(i) \cap N(j)}^{R}+\Gamma_{j, N(i) \cap N(j)}^{T} \\
+\Gamma_{j, N(i) \cap N(j)}^{R}+\max \left(\Gamma_{i, N(i)-N(j)}^{T}, \Gamma_{j, N(j)-N(i)}^{T}\right) \\
\quad+\max \left(\Gamma_{i, N(i)-N(j)}^{R}, \Gamma_{j, N(j)-N(i)}^{R}\right)+\eta_{j i} \\
R_{\max }^{1}(i, j)=\Gamma_{i}^{T}+\Gamma_{i}^{R}+\Gamma_{j}^{T}+\Gamma_{j}^{R}-2 \eta_{i j}-\eta_{j i}
\end{array}
$$

Case $\mathbf{F}>1$ : If $F>1$, then

$$
\begin{aligned}
R_{\min }^{1}(i, j)= & \max \left(\Gamma_{i}^{T}+\Gamma_{i}^{R}-\eta_{i j}, \Gamma_{j}^{T}+\Gamma_{j}^{R}-\eta_{i j}\right)+ \\
& O_{N(i) \cap N(j)} \\
R_{\max }^{1}(i, j)= & \Gamma_{i}^{T}+\Gamma_{i}^{R}+\Gamma_{j}^{T}+\Gamma_{j}^{R}-2 \eta_{i j}-\eta_{j i}
\end{aligned}
$$

where, $O_{N(i) \cap N(j)}$ is the overflow due to nodes $i$ and $j$ transmitting and receiving from common nodes $k$ in set $N(i) \cap N(j)$. This overflow is calculated based on a filling argument; basically the cells corresponding to common node $k$ should be in different time slots. The cells not considered in $R_{\min }^{1}(i, j)$ take up additional cells in a different frequency and need to be considered when calculating 
$C_{l}^{\max }$. Let the number of these cells be $S_{\min }^{1}(i, j)$.

$$
\begin{aligned}
S_{\min }^{1}(i, j)= & R_{\max }^{1}(i, j)-R_{\min }^{1}(i, j) \\
& -\min \left(\Gamma_{i, N(i)-N(j)}^{T}, \Gamma_{j, N(j)-N(i)}^{T}\right) \\
& -\min \left(\Gamma_{i, N(i)-N(j)}^{R}, \Gamma_{j, N(j)-N(i)}^{R}\right)
\end{aligned}
$$

2) Computing Conflict Graph and Cliques for $R_{\min }^{2}(i, j)$ and $R_{\min }^{3}(i, j): R_{\min }^{2}(i, j)$ is the low estimate of the number of cells used by neighbors of $i$ (except $j$ ) to receive traffic from their neighbors (except transmissions from $i$ and $j$ ). This corresponds to finding the maximum of sum of average cells used by those neighboring nodes (except $j$ ) of $i$ that cannot receive simultaneously. Hence we create a conflict graph whose vertices are the links in the neighborhood of $i$ (excluding links with receiver node $j$ ) that receive data from nodes other than $i$ and $j$ and with edges between those links (vertices) that cannot receive simultaneously. From this conflict graph, we find all the maximal cliques and use them to find $R_{\min }^{2}(i, j)$. An edge is drawn between two vertices in the conflict graph, say, links $(k, l)$ and $(m, n)$, if $k=(m$ or $n)$, if $l=(m$ or $n)$, if $n$ is within transmit range of $k$, or if $l$ is within transmit range of $m$. The set of maximal cliques can be found by Bierstone's method [10].

$R_{\min }^{3}(i, j)$ is the maximum of sum of average cells used by neighboring nodes (except $i$ ) of $j$ that cannot transmit simultaneously and is computed in a similar manner to $R_{\min }^{2}(i, j)$ with the conflict graph created from links around node $j$ (excluding links with transmitting node $i$ ) that transmit to nodes other than $i$ and $j$.

3) Computing $R_{\min }^{2}(i, j), R_{\min }^{3}(i, j)$, and $\eta_{\min }^{c o m n}(i, j)$ : The rationale behind computing $R_{\min }^{2}(i, j)$ and $R_{\min }^{3}(i, j)$ is that at least these $R_{\min }^{2}(i, j)+R_{\min }^{3}(i, j)$ number of cells need to be reserved and hence need to be subtracted from available cells when computing $C_{l}^{\max }$. But there could be some common links between the maximal cliques used to compute $R_{\min }^{2}(i, j)$ and $R_{\min }^{3}(i, j)$ that contribute a total of $\eta_{\min }^{\text {comn }}(i, j)$ cells. Let $r_{\min }^{2}(i, j)$ be the sum of the average cells reserved by the links in a maximal clique around node $i$, let $r_{\text {min }}^{3}(i, j)$ be the sum of the average cells reserved by the links in a maximal clique around node $j$, and let $\eta^{\text {comn }}(i, j)$ be the sum of average cells reserved by the common links between $r_{\min }^{2}(i, j)$ and $r_{\min }^{3}(i, j)$. Hence we set $R_{\min }^{2}(i, j), R_{\min }^{3}(i, j)$, and $\eta_{\min }^{\text {comn }}(i, j)$ to be that $r_{\min }^{2}(i, j), r_{\min }^{3}(i, j)$, and $\eta^{c o m n}(i, j)$ respectively that maximizes the sum $r_{\min }^{2}(i, j)+r_{\min }^{3}(i, j)-\eta^{\operatorname{comn}}(i, j)$.

4) Computing $R_{\max }^{2}(i, j), R_{\max }^{3}(i, j)$, and $\eta_{\max }^{c o m n}(i, j)$ :

$$
\begin{aligned}
& R_{\max }^{2}(i, j)=\sum_{k \in\{N(i)-j\}}\left(\Gamma_{k}^{R}-\eta_{i k}-\eta_{j k}\right) \\
& R_{\max }^{3}(i, j)=\sum_{k \in\{N(j)-i\}}\left(\Gamma_{k}^{T}-\eta_{k j}-\eta_{k i}\right)
\end{aligned}
$$

$$
\begin{aligned}
\eta_{\max }^{\text {comn }}(i, j)=\sum_{k \in\{N(j)-i\}} \sum_{m \in\{N(i)-j\}} \eta_{k m} \\
\text { 5) Computing } C_{l}^{\min } \text { and } C_{l}^{\max }: \text { For } F=1, \\
C_{l}^{\min }=\max \left\{0, M-\left(R_{\max }^{1}(i, j)+R_{\max }^{2}(i, j)\right.\right. \\
\left.\left.+R_{\max }^{3}(i, j)-\eta_{\max }^{\text {comn }}(i, j)\right)\right\} \\
C_{l}^{\max }=\max \left\{0, M-\left(R_{\min }^{1}(i, j)+R_{\min }^{2}(i, j)\right.\right. \\
\left.\left.+R_{\min }^{3}(i, j)-\eta_{\min }^{c o m n}(i, j)\right)\right\}
\end{aligned}
$$

For $F>1$,

$$
\begin{aligned}
C_{l}^{\min }= & \max \left\{0, M-R_{\max }^{1}(i, j)-\left[R_{\max }^{2}(i, j)+\right.\right. \\
& \left.\left.R_{\max }^{3}(i, j)-\eta_{\max }^{c o m n}(i, j)-M \times(F-1)\right]^{+}\right\} \\
C_{l}^{\max }= & \max \left\{0, M-R_{\min }^{1}(i, j)-\left[S_{\min }^{1}(i, j)+\right.\right. \\
& R_{\min }^{2}(i, j)+R_{\min }^{3}(i, j)-\eta_{\min }^{c o m n}(i, j)- \\
& \left.M \times(F-1)]^{+}\right\}
\end{aligned}
$$

\section{USAP HARD SCHEDULING THROUGHPUT SENSITIVITIES}

Total throughput $T H\left(\mathbf{C}_{\mathbf{l}}\right)$ for USAP Hard Scheduling is the total cell demands that are not blocked and depends on the vector of free capacities $\mathbf{C}_{\mathbf{l}}$ over all the links $l$, i.e.,

$$
T H\left(\mathbf{C}_{\mathbf{l}}\right)=\sum_{s \in S} \sum_{r=1}^{k_{s}} n_{s} \alpha_{r_{s}} \frac{\nu_{s}}{\mu_{s}}\left(1-L_{r}\right)
$$

where $S$ is the set of all source-destination connections, $k_{s}$ is the total number of routing paths for a connection $s, n_{s}$ is the call demand (number of reserved cells per frame) for connection $s$, and $\alpha_{r_{s}}$ is the fraction of calls that are routed over path $r$ for connection $s$.

For the reduced load approximation of a multi-service loss network, it is possible to analytically calculate the throughput sensitivities using the implied cost formulation (see section 5.7 of [9]). In order to connect to the implied cost formulation, $n_{s}$ is equal to the rate at which a call on route $r$ for connection $s$ earns revenue. Consider adding a single call to route $r$ of connection $s$ in equilibrium. This call is admitted with probability $1-L_{r}$; if admitted the call uses an average of $n_{s} / \mu_{s}$ cells or earns an average revenue of $n_{s} / \mu_{s}$, but reduces the future expected revenue or throughput due to the additional blocking that its presence causes. This expected loss in future revenue or throughput is called the implied cost $\left(c_{r_{s}}\right)$ of route $r$ call of connection $s$. Hence throughput sensitivities are given by:

$$
\begin{aligned}
\frac{\partial}{\partial \alpha_{r_{s}}} T H\left(\mathbf{C}_{\mathbf{l}}\right) & =\nu_{s}\left(1-L_{r}\right)\left(\frac{n_{s}}{\mu_{s}}-c_{r_{s}}\right) \\
\text { where, } c_{r_{s}} & =\frac{1}{\mu_{s}}\left[T H\left(\mathbf{C}_{\mathbf{l}}\right)-T H\left(\mathbf{C}_{\mathbf{l}}-\mathbf{n}_{\mathbf{l r _ { \mathbf { s } }}}\right)\right]
\end{aligned}
$$


and $\mathbf{n}_{\mathbf{l r}_{\mathrm{s}}}$ is a vector specifying the call demand for route $r$ of connection $s$ over all the links $l$. The implied costs are approximated using link independence assumption. Thus the total implied cost for route $r$ of connection $s$ is approximated to be the sum of individual link implied costs along all links $l$ of route $r$ of connection $s$ and is given by:

$$
c_{r_{s}}=\sum_{l \in r_{s}} c_{l r_{s}}
$$

A fixed point approximation procedure is used to find the link implied costs similar to that in [9] (section 5.7). The equations are:

$$
c_{l r_{s}}=\sum_{r_{s^{\prime}}^{\prime} \in R_{l}} \frac{\Delta_{r_{s^{\prime}}^{\prime}} l_{s} \rho_{l, r_{s^{\prime}}^{\prime}}}{\mu_{s}}\left[n_{s^{\prime}}-\mu_{s^{\prime}} \sum_{i \in r_{s^{\prime}}^{\prime}-l} c_{i r_{s^{\prime}}^{\prime}}\right]
$$

where,

$$
\begin{gathered}
\Delta_{r_{s^{\prime}}^{\prime} l r_{s}=\sum_{m=C_{l}^{\text {min }}}^{C_{l}^{\text {max }}}(}\left(P [ C _ { l } = m ] \left\{Q_{r_{s^{\prime}}^{\prime}}\left[m-n_{s} ; \rho_{l, q}, q \in R_{l}\right]\right.\right. \\
\left.\left.-Q_{r_{s^{\prime}}}\left[m ; \rho_{l, q}, q \in R_{l}\right]\right\}\right)
\end{gathered}
$$

Having obtained the throughput sensitivities with respect to routing probabilities, we use the gradient projection method to find the optimal values for routing probabilities to maximize total network throughput.

\section{RESULTS AND VALIDATION}

\section{A. Scenario}

The scenario considered is a fast moving network of 30 vehicles heading towards a rendezvous point. The scenario duration is for 500 seconds with vehicles moving at speeds between $22-60 \mathrm{mph}$. The vehicles start together, then branch into 3 clusters of 10 nodes each due to obstructions (2 steep hillocks), and finally rejoin (see figure 3 ). Two Aerial Platforms (APs) are used to maintain communication connectivity when the clusters become disconnected. The number and location of the APs are determined by a fast Deterministic Annealing algorithm [11]. From 0-30s, the ground nodes move together forming a connected network. From $30-420$ s, the nodes form 3 clusters as shown in figure 3 with clusters 1 (nodes 0-9) and 3 (nodes 20-29) going around the hills. The clusters start to lose communication connectivity around $75 \mathrm{~s}$, then become disconnected from each other, and finally reconnect around 400s. APs are brought in to provide communication connectivity between the otherwise disconnected clusters from 75-400s.

The scenario is specified every 5 seconds (the ground nodes move an average of 100 meters in 5s). At every 5 second interval, the ground node positions, the traffic demands (offered load) \& routes between source-destination

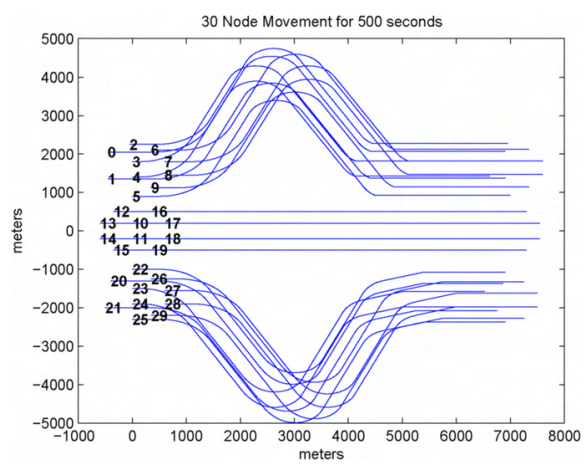

Fig. 3. 30 node movement for 500 seconds

pairs, and the environment conditions are input to the USAP Hard Scheduling performance models. All ground nodes and APs have identical omni-directional radios with receiver sensitivity of $-95 \mathrm{dBm}$, receiver threshold of $10 \mathrm{~dB}$, and transmit power of $5 \mathrm{~W}$. The environment is modeled as a fading channel with a $1 / R^{\alpha}$ power attenuation. The radio specification and the path loss exponent $\alpha$ together determine a maximum connectivity distance between nodes. $\alpha$ is taken to be 4.5 between ground nodes, 3.9 between ground and aerial nodes, and 3.0 between the aerial nodes. This results in a maximum connectivity distance of $857 \mathrm{~m}$ between ground nodes, $2423 \mathrm{~m}$ between ground-aerial nodes, and $25099 \mathrm{~m}$ between aerial nodes. The maximum channel rate between any two nodes is set to $1 \mathrm{Mbps}$.

There are 17 source-destination connection pairs chosen in this scenario. The traffic between each source-destination pair is routed via the first $K$ shortest distance paths. All the connections have holding time of 2 minutes. There are 13 intra-cluster connections (4 each in cluster 1 and 2; and 5 in cluster 3 ) each of which have call arrival rate of 2.5 calls/minute and with $K$, the number of paths per connection, equal to 2 or 3 . The remaining 4 connections span clusters with $K$ ranging from 2 to 4 paths and call arrival rate ranging from $0.5-2.5$ calls/minute. Connection 11 between source node 20 and destination node 0 is the longest connection (with $K=4$ ).

\section{B. USAP Parameters}

The USAP frame period is $125 \mathrm{~ms}$. The number of frequency channels $(F)$ is set to 2 and the number of reservation time slots $(M)$ is set to 25 . Only half of the USAP frame period is used for reservation slots. Based on the sum capacity of all channels, $M, F$, and the fraction of frame period used for reservation slots, 1250 bits can be carried per reservation cell. Hence for a connection to have a call demand $\left(n_{r}\right)$ of 1 reservation slot per frame, the call demand rate (for e.g., the voice coder rate) is $10 \mathrm{kbps}$. We assume that the voice coder rate is $10 \mathrm{kbps}$ (hence voice calls use 1 reservation cell per frame) and the voice coder 


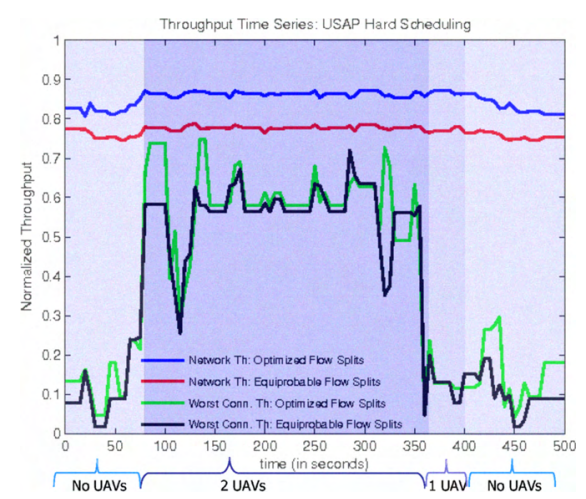

Fig. 4. Throughput Time Series: USAP Hard Scheduling

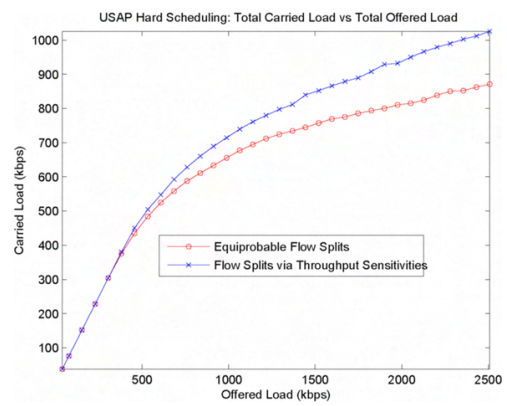

Fig. 5. Total Carried Load vs Offered Load: USAP Hard Scheduling

frame period is $125 \mathrm{~ms}$. All chosen source-destination pairs have a call demand of 1 reservation slot per frame.

\section{Hard Scheduling With Capacity Estimation Model}

We run the entire scenario first with equiprobable flow splits among the various paths of a connection and then with flow splits optimized using throughput sensitivities (section IV). Figure 4 shows the variation of total throughput and connection 11 throughput (worst connection throughput) for the two cases. Note the increase in total throughput for flow splits chosen to maximize total throughput.

To find out effects of offered load on throughput, we ran the scenario at time snapshot 0 but with all connection offered loads scaled by a common factor $\delta$. Figure 5 shows the effect of offered load on total throughput for equiprobable flow splits and flow splits chosen to maximize throughput. The total throughput in both cases saturates to some maximum value which is the maximum total capacity that the reservation based system can carry.

\section{Validation}

We developed a simulation of USAP Hard Scheduling and use it to validate the reduced load loss network models. Table I compares the total throughput between simulation and reduced loss models for various load scaling factors. Although the total throughputs are close for both the simulation and models, the individual connection throughput varies quite a bit. Figure 6 compares the throughput
TABLE I

Total Throughrut: Simulation VS Models

\begin{tabular}{|c|c|c|c|c|}
\hline Load Factor & 0.5 & 1.0 & 1.5 & 2.0 \\
\hline Sim Th & 0.9742 & 0.8011 & 0.6370 & 0.5227 \\
\hline Model Th & 0.9867 & 0.7738 & 0.6092 & 0.4983 \\
\hline
\end{tabular}

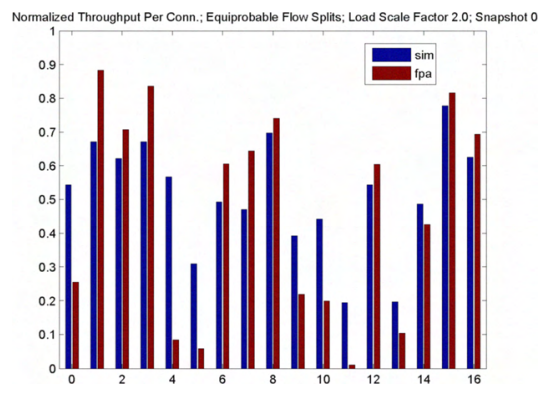

Fig. 6. USAP Hard Scheduling: Simulation VS Models

TABLE II

Total Throughrut: Simulation VS Models With Simulation FREE CAPACITY PMF

\begin{tabular}{|c|c|c|c|c|}
\hline Load Factor & 0.5 & 1.0 & 1.5 & 2.0 \\
\hline Sim Th & 0.9742 & 0.8011 & 0.6370 & 0.5227 \\
\hline $\begin{array}{l}\text { ModeI Th (with sim's free } \\
\text { capacity pmf) }\end{array}$ & 0.9800 & 0.8019 & 0.6328 & 0.5171 \\
\hline
\end{tabular}

of each connection between simulation and reduced load models (with reduced link capacity model as per section III-A) . We see that there is a large mismatch for some connections (connection $0,4,5,9,10,11$ ). To find out if the mismatch is due to the reduced link capacity model used, we ran the reduced load loss network models using the reduced link capacity pmf obtained using simulation. Table II shows good match for the total throughput at various load scaling factors between simulation and reduced load loss network models using simulation's free capacity pmf. Figure 7 compares the individual connection throughput obtained in this manner against simulation. We see that the throughput matches very well for all connections. This leads us to use the reduced link capacity pmf obtained through simulation along with our reduced load loss network models and throughput sensitivity formulas (section IV) in order to optimize total throughput. The next section presents results of using this simulation based optimization method.

\section{E. Hard Scheduling With Simulation Based Capacity Esti- mation}

Since using simulation based reduced link capacity pmf along with reduced load loss network models results in perfect match with simulation, we use simulation along with our reduced load models to obtain throughput sensitivities (section IV)) and then optimize total throughput. Figure 8 shows the variation of total throughput and worst connection throughput (i.e., connection 11) for the two cases of using equiprobable flow splits and flow splits chosen to 


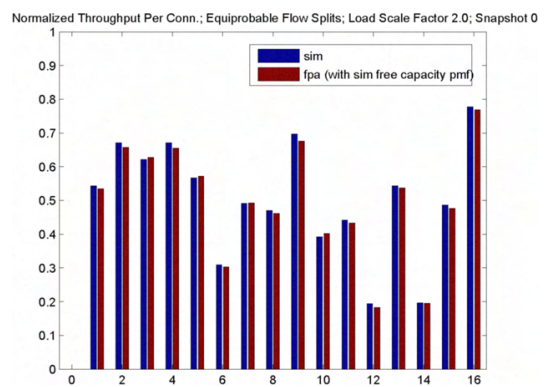

Fig. 7. USAP Hard Scheduling: Simulation VS Model with capacity estimation pmf obtained from simulation

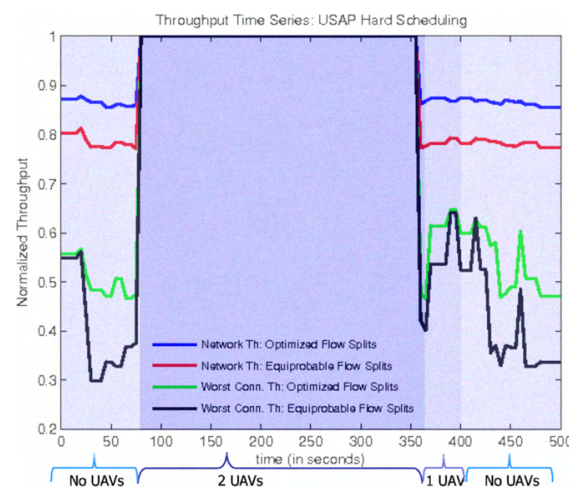

Fig. 8. Throughput Time Series: USAP Hard Scheduling Model with simulation based capacity estimation

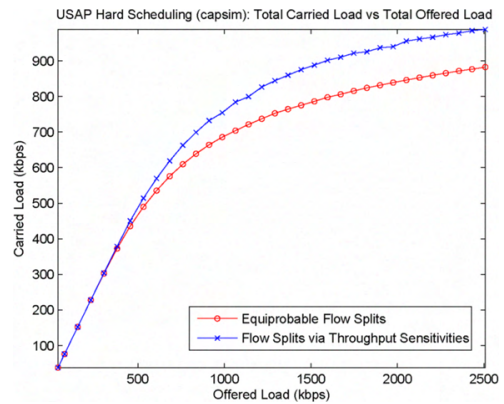

Fig. 9. Total Carried Load vs Offered Load: USAP Hard Scheduling Model with simulation based capacity estimation

optimize throughput. The total throughput increases with the flow splits chosen to maximize total throughput.

Figure 9 shows the effect of varying offered load (at time snapshot 0 ) on total throughput for equiprobable flow splits and flow splits chosen to maximize throughput. The total throughput in both cases saturates to some maximum value which is the maximum total capacity that the reservation based system can support.

\section{CONCLUSIONS}

We have developed and evaluated models based on reduced load loss networks with reduced link capacity to estimate various performance metrics for unicast traffic using hard scheduling mode of reservation based protocol
USAP in MANETs. We compare our results against simulation and find that substituting the reduced link capacity estimation with the link capacity pmf from simulation gives good results. We therefore need to improve our reduced link capacity estimation in order to obtain a good model for USAP Hard Scheduling. We further use the reduced load loss network models to analytically obtain throughput sensitivities and use this to optimize total throughput both with our reduced link capacity model and with link capacity pmf obtained through simulation. Thus we use simulation to obtain link capacity pmf but use the reduced loss models to provide a search direction in order to design the routing probabilities to maximize network throughput.

\section{ACKNOWLEDGMENT}

This work was supported by SBIR Phase II contract W15P7T-07-C-P613 from U.S.Army CECOM to AIMS Inc.

\section{REFERENCES}

[1] C.D. Young, "USAP: a unifying dynamic distributed multichannel TDMA slot assignment protocol," In Proceedings of MILCOM 1996, Oct. 1996.

[2] F.P Kelly, "Loss networks," Annals of Applied Probability, 1(3):319-378, Aug 1991.

[3] F.P Kelly, "Blocking probabilities in large circuit switched networks," Advances in Applied Probability, 18(2):473-505, June 1986.

[4] J.A. Morrison, D. Mitra, and K.G. Ramakrishnan, "ATM network design and optimization: A multirate loss network framework," IEEE/ACM Transactions in Networking, 4(4):531-543, Aug 1996.

[5] A. Kashper, S. Chung, and K.W. Ross, "Computing approximate blocking probabilities with state- dependent routing," IEEE/ACM Transactions in Networking, 1(1):105-115, Feb 1993.

[6] R. Srikanth and A.G. Greenberg, "Computational techniques for accurate performance evaluation of multirate, multihop communications networks," IEEE J. Sel. Areas Communications, 5(2):266-277, Feb 1997.

[7] J.S Baras and M. Liu, "Fixed point approximation for multirate multihop loss networks with adaptive routing," IEEE/ACM Trans Networking, 12(2):361-374, April 2004.

[8] C.D. Young, "The Mobile Data Link (MDL) of the Joint Tactical Radio System Wideband Networking Waveform," In Proceedings of MILCOM 2006, 23-25 Oct 2006.

[9] Keith W. Ross. "Multiservice Loss Models for Broadband Telecommunication Networks," Springer Telecommunications Networks and Computer Systems, 1995.

[10] Gordon D. Mulligan and D. G. Corneil, "Corrections to Bierstone's Algorithm for Generating Cliques," Journal of the ACM, Vol. 19, No.2, April 1972, pp. 244-247.

[11] S. Perumal, J. S. Baras, C.J. Graff, and D.G. Yee, "Aerial Platform Placement Algorithms to Satisfy Connectivity, Capacity and Survivability Constraints in Wireless Ad-hoc Networks," In Proceedings of MILCOM 2008, 16-19 Nov 2008. 